\title{
Potencial acaricida do extrato de folhas de Xylopia emarginata Mart. contra larvas de Rhipicephalus microplus
}

\author{
Juliana Pimenta Cruz ${ }^{1 *}$; Viviane de Oliveira Vasconcelos ${ }^{2}$; Patrícia Conceição Medeiros ${ }^{3}$; João Carlos Figueire- \\ do $^{4}$; Yule Roberta Ferreira Nunes ${ }^{5}$; Eduardo Robson Duarte ${ }^{6}$; Ana Paula Venuto
}

DOI: https://doi.org/10.35699/2447-6218.2021.34636

\begin{abstract}
Resumo
O objetivo desse trabalho foi avaliar a ação carrapaticida in vitro do extrato etanólico (EE) de folhas de Xylopia emarginata Mart. com diferentes teores de taninos sobre larvas de R. microplus. Para isso, as folhas de X. emarginata foram coletas mensalmente durante o período de 12 meses para avaliar a produção de taninos através da técnica de solvólise catalisada por butanol/HCl. Para avaliar a eficácia do EE, foi realizado o teste do pacote de larvas (TPL) com leitura no intervalo de 24 horas de incubação utilizando as concentrações de 25 a $100 \mathrm{mg} \mathrm{mL}^{-1}$, o controle negativo contendo água destilada e o controle positivo contendo carrapaticida químico. Observou-se que o EE contendo 5,9\% de teor de taninos promoveu 80,44\% de mortalidade na concentração de $100 \mathrm{mg} \mathrm{mL}^{-1}$. O EE contendo 13,2\% de teor de taninos promoveu $97,43 \%$ de mortalidade a $100 \mathrm{mg} \mathrm{mL}^{-1}$. Dessa forma, constatou-se alta atividade larvicida dos extratos etanólicos de folhas de $X$. emarginata sobre larvas de $R$. microplus e essa atividade pode estar relacionada aos teores de taninos.
\end{abstract}

Palavras-chave: Annonaceae. Carrapato bovino. Fitoterapia. Taninos.

\section{Acaricide potential of Xylopia emarginata Mart. leaf extract against Rhipicephalus microplus larvae}

\begin{abstract}
The objective of this work was to evaluate the in vitro carrapaticidal action of ethanol extract (EE) of Xylopia emarginata leaves Mart.com different tannin contents on $R$. microplus larvae. For this, as leaves of $X$. emarginata were collected monthly during the period of 12 months to evaluate the production of tannins through the solveylysis technique catalyzed by butanol/HCl. To evaluate the efficacy of EE, the larvae bundle test (TPL) was performed with reading in the interval of 24 hours of incubation using concentrations of 25 to $100 \mathrm{mg} \mathrm{mL}^{-1}$, the negative control containing distilled water and the positive control containing chemical carrapaticide. It was observed that the EE containing 5.9\% tannin content promoted $80.44 \%$ mortality at the concentration of $100 \mathrm{mg} \mathrm{mL}^{-1}$. The EE containing $13.2 \%$ tannin
\end{abstract}

${ }^{1}$ Universidade Estadual de Montes Claros. Montes Claros, MG. Brasil. https://orcid.org/0000-0001-5115-3136

${ }^{2}$ Universidade Estadual de Montes Claros. Montes Claros, MG. Brasil. https://orcid.org/0000-0001-5126-3124

${ }^{3}$ Instituto Federal de Minas Gerais. Januária, MG. Brasil.

https://orcid.org/0000-0002-2949-3940

${ }^{4}$ Universidade Estadual de Montes Claros. Montes Claros, MG. Brasil. https://orcid.org/0000-0001-6453-8684

${ }^{5}$ Universidade Estadual de Montes Claros. Montes Claros, MG. Brasil. https://orcid.org/0000-0003-3328-7506

${ }^{6}$ Universidade Federal de Minas Gerais. Montes Claros, MG. Brasil. https://orcid.org/0000-0002-2205-9412

${ }^{7}$ Universidade Estadual de Montes Claros. Montes Claros, MG. Brasil. https://orcid.org/0000-0002-3103-7063

*Autor para correspondência: JulianaPimenta97@outlook.com

Recebido para publicação em 25 de Junho de 2021. Aceito para publicação 30 de Junho de 2021 e-ISSN: 2447-6218 / ISSN: 2447-6218. Atribuição CC BY. 
content promoted $97.43 \%$ mortality at $100 \mathrm{mg} \mathrm{mL}^{-1}$. Thus, high larvicidal activity of ethanol extracts of leaves of $X$. emarginata on Larvae of $R$. microplus was verified and this activity may be related to tannin contents.

Keywords: Annonaceae. Bovine tick. Phytotherapy. Tannins.

\section{Introdução}

O Rhipicephalus microplus representa um dos ectoparasitas mais importantes para a bovinocultura. Esse carrapato é responsável por elevadas perdas econômicas na cadeia produtora de leite, carne e couro, podendo ocasionar o óbito (Roy et al., 2017). Além disso, as hemoparasitoses transmitidas por esse vetor compõe um complexo de enfermidades de importância em saúde pública como a babesiose humana (Hajdulek et al., 2013).

O principal método de controle desse parasito é o químico. Entretanto, o uso indiscrimidado dos produtos carrapaticidas tem favorecido a resistência, inviabilizando assim o controle efetivo, com aumento de risco de contaminação ambiental e os produtos finais de origem animal como carne e leite (Guerrero et al., 2012). A busca por métodos de controle alternativos para o carrapato é uma estratégia fundamental para a criação de bovinos em condições tropicais (Webster et al., 2015).

Muitos metabólitos secundários de plantas têm sido utilizados no controle alternativo para minimizar os impactos causados pelos produtos químicos (Wiesbrook, 2004). O bioma Cerrado possui ampla diversidade de flora contendo compostos e moléculas importantes com principio ativos que podem ser utilizados no controle de ectoparasitos (Bahia et al., 2009). A família Annonaceae é abundante nesse bioma. O gênero Xylopia, pertencente a essa familia (Marimon-Junior e Haridasan, 2005) é reconhecido por apresentar propriedades antimicrobiana, acaricida, inseticida e nematicida (Konning et al., 2004), entretanto ainda não é conhecida sua atividade contra carrapatos. Uma das espécies identificadas no Brasil é Xylopia emarginata Mart. conhecida como "pindaíba-reta", que pode ser encontrada em lugares brejosos e em matas ciliares, principalmente nos estados da Bahia, Minas Gerais, Goiás, Mato Grosso do Sul e São Paulo (Lorenzi, 1992).

Neste estudo, o objetivo foi avaliar o efeito carrapaticida in vitro do extrato etanólico das folhas de $X y$ lopia emargianata contendo diferentes teores de taninos condensados contra larvas de Rhipicephalus microplus.

\section{Material e métódos}

\section{Obtenção dos extratos etanólicos}

O material vegetal foi composto por folhas de $X$. emarginata coletadas no município de Bonito de Minas, no norte de Minas Gerais, na vereda Almescla (coordenadas $45^{\circ} 95^{\prime} \mathrm{W}, 15^{\circ} 88^{\prime} \mathrm{Se} 43^{\circ} 95^{\prime} \mathrm{W}, 14^{\circ} 40^{\prime \prime} \mathrm{S}$ ). As amostras foram coletadas mensalmente no período de junho e setembro de 2018. As exsicatas foram depositadas no Herbário Montes Claros, voucher 6774 e a espécie foi identificada considerando as caracteristicas descritas em Lorenzi (1992).

As folhas foram selecionadas e foram descartadas aquelas com deteriorações. O material foi desidratado em estufa a $40^{\circ} \mathrm{C}$ por 72 horas, e trituradas com o auxílio de um triturador (Di Grano MDR - 302, Marca, estado, pais). O pó foi armazenado em temperatura ambiente $\mathrm{e}$ em sacos de papel para evitar incidência de luz.

O extrato etanólico (EE) foi obtido por meio da submersão de $100 \mathrm{~g}$ de material vegetal em $1000 \mathrm{~mL}$ de etanol PA (álcool etílico), em recipientes de vidro âmbar, conservados em local escuro e por sete dias. Os extratos foram filtrados em funil,com gaze e algodão e levados à estufa de circulação forçada de ar a $40^{\circ} \mathrm{C}$, até a obtenção de peso constante (Nery et al., 2010). Os extratos secos foram removidos e armazenados em frascos escuros e as subalíquotas foram submetidas ao determinador de matéria seca (Association of Official Analytical Chemists - AOAC, 1990) (mg mL $\left.{ }^{-1}\right)$ a $105^{\circ} \mathrm{C}$ para cálculo das concentrações avaliadas.

A deteminação dos teores totais de taninos condensados (proantocianidinas) foi avaliada ao longo do período amostral, de acordo com Medeiros et al. (2019), após solvólise catalisada por ácido com n-BuOH/HCl $37 \%$ (95:5), seguindo metodologia descrita por Hiermann et al. (1986). Constatou-se que o EE de folhas coletadas em junho e setembro apresentaram $13,2 \%$ e $5,9 \%$ de teores de taninos condensados, respectivamente (Medeiros et al., 2019).

Eficácia dos extratos contra larvas de $R$. microplus: teste do pacote de larvas (TPL)

Para o experimento, foram utilizadas larvas de 15 a 28 dias pós-eclosão, provenientes de infestação natural em bovinos provenientes de uma propriedade rural de Bocaiúva-MG. Esses animais não tiveram contato com carrapaticida por aproximadamente 30 dias.

Os extratos foram avaliados nas concentrações 25, 50, 75 e $100 \mathrm{mg} \mathrm{mL}^{-1}$. Como controle negativo foi utilizado água purificada e a solução de Cipermetrina a $0,025 \mathrm{mg} \mathrm{mL}^{-1}$ (Butox MSD Saúde Animal, São Paulo Brasil) como controle positivo. Os procedimentos foram realizados em quatro repetições. 
Os testes foram realizados de acordo com Stone e Haydock (1962), inserindo-se aproximadamente 50 larvas em pacotes confeccionados de papel filtro (Whatmann no1) de 6 × $6 \mathrm{~cm}$ para cada repetição. Posteriormente, os pacotes foram vedados com grampos e impregnados pelas soluções. Cada grupo de repetições foi depositado em placas de Petri. As larvas incubadas foram acondicionas em estufa a $28^{\circ} \mathrm{C}$ e aproximadamente $70 \%$ de umidade relativa por 24 horas. Após esse período os pacotes foram abertos sobre uma superfície branca, e as larvas vivas e mortas foram quantificadas.

\section{Análises estatísticas}

O estudo foi realizado em delineamento inteiramente casualisado comparando-se com o controle negativo contendo água e o controle positivo contendo cipermetrina.

O número relativo de larvas mortas sobre o número total de larvas foi utilizado para análise de variância e as médias comparadas pelo teste de Scott-Knott com 5\% de significância em delineamento inteiramente casualizado. As concentrações letais para matar $90 \%$ de larvas (CL90) foram estimadas pela análise de regressão probit do pacote estatístico Saeg 9.1.

\section{Resultados e discussão}

Os resultados consistem na primeira verificação da atividade acaricidade de EE de folhas de $X$. emarginata frente a larvas de $R$. microplus. O EE extraído de folhas coletadas durante o mês de setembro $(5,9 \%$ de teor de taninos) promoveu $80,44 \%$ de mortalidade larval na concentração $100 \mathrm{mg} \mathrm{mL}^{-1}(\mathrm{p} \leq 0,05)$ (Tabela 1). A CL90 para esse extrato foi de $44,08 \mathrm{mg} \mathrm{mL}^{-1}$ (Figura 1a). O EE extraído de folhas coletadas do mês de junho $(13,2 \%$ de teor de taninos condensados) promoveu taxas de mortalidade larval entre 96,43 a 97,43\% nas concentrações de 75 e $100 \mathrm{mg} \mathrm{mL}^{-1}$ respectivamente e que foram superiores ao controle contendo água destilada $(\mathrm{p} \leq 0,05)$ (Tabela 1). ACL90 para esse extrato foi de $41,54 \mathrm{mg} \mathrm{mL}^{-1}$ (Figura $1 \mathrm{~b})$.

Tabela 1 - Mortalidade larval de Rhipicephalus microplus tratados com extrato etanólico de folhas de Xylopia emarginata com $5,9 \%$ e $13,2 \%$ deteor de taninos condensados.

\begin{tabular}{lcc}
\hline Tratamentos & Taninos condensados & Taninos condensados \\
\hline (mg mL-1) & $(5,9 \%)$ & $(13,2 \%)$ \\
100 & $80,44 \mathrm{Bb}$ & $97,43 \mathrm{Aa}$ \\
75 & $77,28 \mathrm{Bb}$ & $96,43 \mathrm{Aa}$ \\
50 & $74,23 \mathrm{Ba}$ & $75,49 \mathrm{Ba}$ \\
25 & $7,97 \mathrm{Cb}$ & $51,24 \mathrm{Ca}$ \\
Água estéril & $0 \mathrm{Da}$ & $0 \mathrm{Da}$ \\
Cipermetrina* & $100 \mathrm{Aa}$ & $100 \mathrm{Aa}$ \\
CV(\%)** & 15 & 10 \\
* Cipermetrina 0,025 mg /mL (ButoxMSD Saúde Animal, São Paulo Brasil). & \\
**Coeficiente de variação (\%) \\
$\begin{array}{l}\text { Letras maiúsculas distintas indicam diferença significativa (p }<0,05) \text { entre os tratamentos e minúsculas indicam diferença significativa (p < 0,05) } \\
\text { entre as diferentes concentrações de tanino, por teste de Scott-Knott a } 5 \% .\end{array}$
\end{tabular}

Ao analisar a eficácia entre os diferentes extratos em relação aos teores de taninos, observou-se diferença significativa nas concentrações de 100, 75 e $25 \mathrm{mg} \mathrm{mL}^{-1}$ dos extratos contenho $13,2 \%$ de teor de tanino, sendo estatisticamente superior a eficácia dos extratos com $5,9 \%$ de teor de taninos.

Sabe- se que os taninos condensados são importantes no controle de parasitos pois essas substâncias são classificadas como redutores digestivos, com efeito proporcional à concentração (Monteiro et al., 2011). Reduzem significativamente o crescimento e a sobrevivência de artrópodes, uma vez que inativam enzimas digestivas e criam um complexo de taninos-proteínas de difícil digestão (Mello e Silva-Filho, 2002).
Segundo Fernández -Salas (2011), extratos etanólicos de plantas ricas em tanino como Acacia pennatula, Piscidia piscipula, Leucaena aleucocephala e Lysiloma latisiliquum representam alternativa promissora para o controle de R. microplus. Entretanto, Vasconcelos et al. (2018) observaram que presença de níveis baixos de taninos em extratos etanólicos de Ximenia americana, Piptadenia viridiflora e Schinopsis brasiliensis também promoveram eficácia acaricida contra teleóginas de Dermacentor nitens nas concentrações de 100 e $150 \mathrm{mg} \mathrm{mL}^{-1}$, indicando que outros metabólitos tambem poderiam participar com o efeito acaricida.

O potencial acaricida de Annonacea pôde ser comprovado em estudo de Broglio-Micheletti (2009), que avaliou o extrato etanólico das sementes de Annona 
Cruz, J. P. et al.

muricata na concentração de $2 \%$ verificando $100 \%$ de eficácia para mortalidade de teleóginas de $R$. microplus. O extrato aquoso da casca do fruto de Annona squamosa apresentou atividade larvicida contra $R$. microplus, sendo observadas mortalidades de 92 e 100\% nas concentrações de 1.500 e 2.000 ppm, respectivamente (Madhumitha et al., 2012).

Figura 1 - Porcentagerm de sobrevivência de larvas de Riphicephalus microplus em função da concentração do extrato etanólico de folhas de Xylopia emarginata com 5,9\% (a) e 13,2\% (b) de teor de tanino.

\section{(A)}

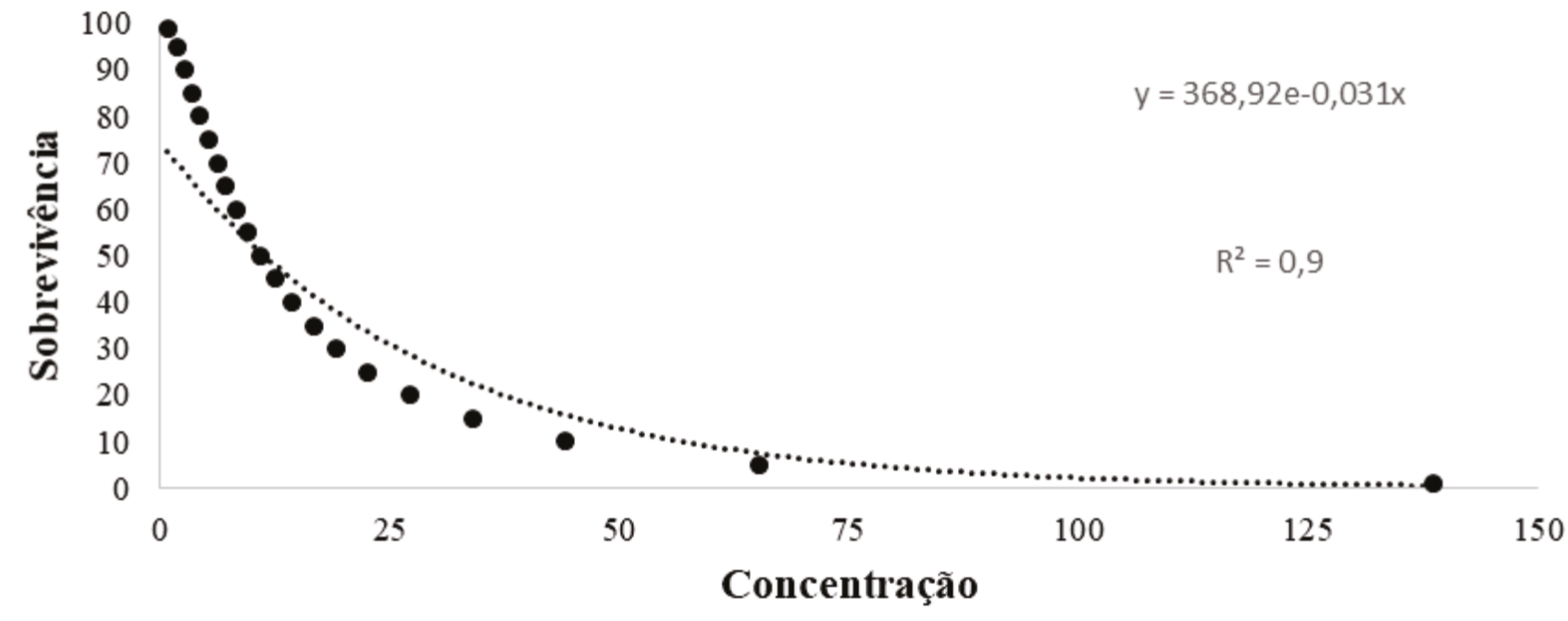

(B)

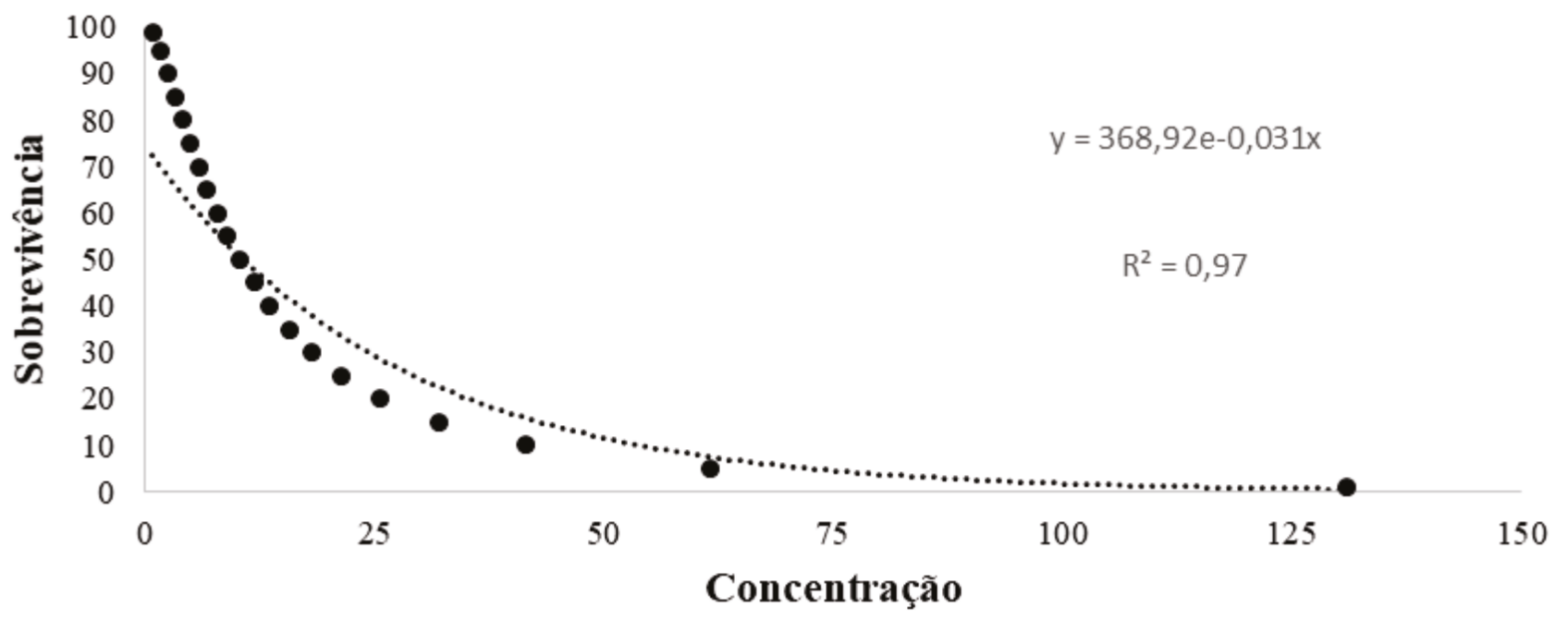

Catto et al. (2009), observou em estudo, atividade acaricida elevada dos extratos etanólicos da raiz de Anonna dioica, que apresentou efeito acaricida de 95\% e $98,7 \%$ nas concentrações de 5\% e 20\%. Além disso, óleos essenciais obtidos a partir de frutos e folhas de Xylopia sericea demonstraram atividade acaricida sobre larvas de Tetranychus urticae com eficácia de $95 \%$ a $10 \mathrm{mg} / \mathrm{mL}$ (Pontes et al., 2007).

Deste modo, a espécie $X$. emarginata pode ser incluída como potencial candidata no programa de controle integrado de $R$. microplus, aumentando a possibilidade de obter produtos naturais como alternativas aos acaricidas sintéticos. Futuros estudos devem ser conduzidos com o objetivo de testar o extrato em fêmeas ingurgitadas, bem como identificar os componentes acaricidas presentes que possam ser utilizados em programa de controle de carrapatos. Além disso, devem ser avaliada também a influência do teor de taninos na mortalidade de fêmeas adultas de $R$. microplus para elucidar a sua influência em diferentes fases de crescimento dessa espécie. 
Potencial acaricida do extrato de folhas de Xylopia emarginata Mart. contra larvas de Rhipicephalus microplus

\section{Conclusão}

Os extratos etanólicos obtidos de folhas de Xylopia emarginata apresentam potencial para o controle de larvas de Rhipicephalus microplus, sendo que, o extrato com maior teor de teor de tanino apresentou eficácia mais elevada no controle dessas larvas.

\section{Agradecimentos}

Esta pesquisa foi financiada pelo Programa de Pesquisa Ecológica de Longa Duração (PELD-VERE) do Conselho Nacional de Desenvolvimento Científico e Tecnológico (CNPq 441440/2016-9; 441583/2020-2; 308877/2019-5), Coordenação de Aperfeiçoamento de Pessoal de Nível Superior (CAPES 88887.136273/201700), Fundação de Amparo à Pesquisa do Estado de Minas Gerais (FAPEMIG APQ-04816-17; APQ-02983-17; CRA-PPM-00539-18), Ministério da Ciência, Tecnologia e Inovações, Programa de pós graduação em botânica aplicada (PPGBOT-Unimontes).

\section{Referências}

Bahia, T. O; Luz, G. R; Veloso, M. D. M; Nunes, Y. R. F; Neves, W. V; Braga, L. L; Lima, P. C. V. 2009. Veredas na APA do Rio Pandeiros: importância, impactos ambientais e perspectivas. MG. Biota, 2:.3-13.

Broglio-Michelleti S. M. F. 2009. Extratos de plantas no controle de Rhipicephalus (Boophilus) microplus (Canestrini, 1887) (Acari: Ixodidae) em laboratório. Revista Brasileira de Parasitologia Veterinária (Online), 18: 44-48, Disponível em: https://www.scielo.br/j/rbpv/a/ VdfLqhhshVjjKKR5yVRVr7B/?lang=pt Acesso em: 28 ago. 2018.

Catto, J. B; Bianchin, I; Saito, M. L. 2009. Efeito acaricida in vitro de extratos de plantas do Pantanal no carrapato de bovinos, Rhipicephalus (Boophilus) microplus. Embrapa Gado de Corte, Boletim de Pesquisa e Desenvolvimento, Campo Grande, MS.

Fernández-Salas, A; Alonso-Díaz, M. A; Acosta-Rodrígues, R; TorresAcosta, J. F; Sandoval-Castro, C. A; Rodríguez-Vivas, R. I. 2011. In vitro acaricidal effect of tannin-rich plants against the cattle tick Rhipicephalus (Boophilus) microplus (Acari: Ixodidae). Veterinary Parasitology, 175:113-118.

Guerrero, F. D; Lovis, L; Martins, J. R. 2012. Acaricide resistance mechanisms in Rhipicephalus (Boophilus) microplus. Revista Brasileira de Parasitologia Veterinária, Jaboticabal, 21:1-6.

Hajdulek, O; Šíma, R; Ayllón, N; Jalovecká, M; Perner, J; Fuente, J; Kopáček1, L. 2013. Interaction of the tick immune system with transmitted pathogens. Frontiers in Cellular and Infection Microbiology, 3: 3-26.

Hiermann, A; Kartnig, T. H; Azzam, S. E. 1986. Beitragzur quantitative Best immung der Procyanidine in Crataegus. Scientia Pharmaceutica, 54: 331-337.

Konning, G. H; Ayare, C; Enninson, B. 2004. Antimicrobial activity of some medicinal plants from Ghana. Fitoterapia, 75: 65-67.

Lorenzi, H. 1992. Árvores brasileiras: manual de identificação e cultivo de plantas arbóreas do Brasil. 2. ed.Nova Odessa: Instituto Plantarum.

Marimon-Junior, B. H; Haridasan, M. 2005. Comparação da vegetação arbórea e características edáficas de um cerradão e um cerrado sensu stricto em áreas adjacentes sobre solo distrófico no leste de Mato Grosso, Brasil. Acta Botânica Brasílica, 19: 915-928.

Madhumitha, G; Rajakuma, G; Roopan, S. M; Rahuman, A . A; Priya, K. M; Saral, A. M; Khan, F. R. N; Khanna, V. G; Velayutham, K; Kamaraj, C; Elang, G. 2012. Acaricidal, insecticidal, and larvicidal efficacy of fruit peel aqueous extract of Annona squamosa and its compounds against blood-feeding parasites. Parasitology Research, 111: 2189-2199.
Medeiros, P. C. 2019. Respostas sazonais fenológicas e do teor de taninos condensados de Xylopia emarginata Mart. (Annonaceae) em uma vereda no Norte de Minas Gerais, Brasil, Universidade Estadual de Montes Claros, Minas Gerais, Dissertação de mestrado Disponível em: http://www.ppgb.unimontes.br/index.php/producoes/dissertacoes.

Mello, M. O; Silva-Filho, M. C. 2002. Plant-insect interactions: an evolutionary arms race between two distinct defense mechanism. Brazilian Journal of Plant Physiology, 14 :71-81.

Monteiro, M. V. B.; Bevilaqua, C. M. L.; Morais, S. M.; Machado, L. K. A.; Camurça Vasconcelos, A. L. F; Campello, C. C.; Ribeiro, W. L. C.; Mesquita, M. A. 2011. Anthelmintic activity of Jatropha curcas L. seedson Haemonchus contortus. Veterinary Parasitology, 182: 259-263.

Nery, P. S; Nogueira, F. A; Martins, E. R; Duarte, E. R. 2010. Effects of Anacardium humile leaf extracts on the developmentof gastrointestinal nematode larvae of sheep. Veterinary Parasitology, 171:361-364.

Pontes, W. J; Oliveira, J. C. S; Câmara, C. A. G; Gondim Júnior, M. G. C; Oliveira, J. V; Schwartz, M. O. E. 2007. Atividade acaricida dos óleos de essências de folhas e frutos de Xylopia sobre o ácaro rajado (Tetranychus urticae Koch). Quimica Nova, São Paulo, 30: 838-841.

Roy, B. C; Krücken, J; Ahmed, J. S; Majumder, S; Baumann, M. P; Clausen, P. H; Nijhof, A. M. 2017. Molecular identification of tickborne pathogens infecting cattle in Mymensingh district of Bangladesh reveals emerging species of Anaplasma and Babesia. Transboundary and Emerging Diseases, 65: 231-242.

Stone, B. F; Haydock, K. P. 1962. A method for measuring the acaricide susceptibility of the cattle tick Boophilus microplus, Bulletin of Entomological Research, 53: 563-578.

Vasconcelos, V. O; Costa, E. G. L; Moreira, V. R; Morais-Costa, F; Duarte, E. R. 2018. Efficacy of plants extracts from the Cerrado against adult female of Dermacentor nitens (Acari: Ixodidae). Experimental and Applied Acarology, 75: 419-427.

Webster, A; Reck, J; Santi, L; Souza, U. A; Dall'Agnol, B; Klafke, G. M; Beys-da-Silva, W. O; Martins, J. R; Schrank, A. 2015. Integrated control of an acaricide resistant strain of the cattle tick Rhipicephalus microplus by applying Metarhizium anisopliae associated with cypermethrin and chlorpyriphos under field conditions. Veterinary Parasitology, 207: 302-308, 2015. DOI: 10.1016/j. vetpar.2014.11.021.

Wiesbrook, M. L. 2004. Natural indeed: Are natural insecticides safer and better than conventional insecticides? Illinois Pesticide Review, Urbana, 17:1-8. 